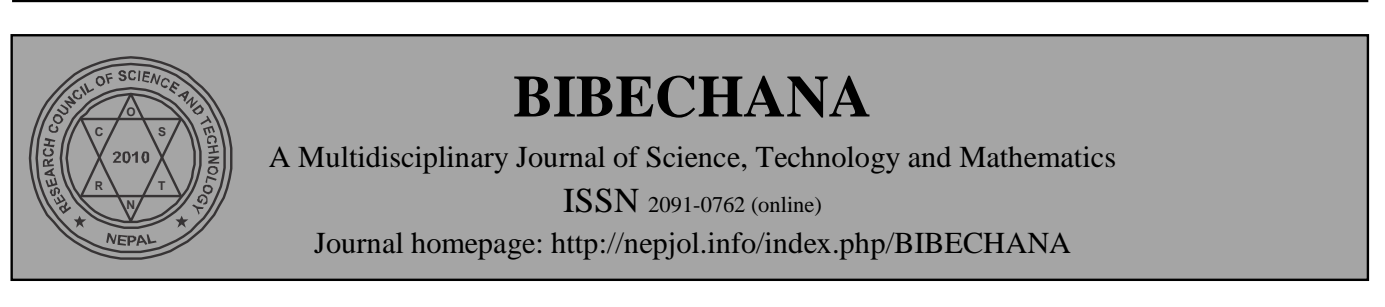

\title{
Dark Matter and Dark Energy in the Universe
}

\author{
B. C. Paul* \\ Department of Physics, University of North Bengal, Darjeeling, 734 013, India \\ Email: bcpaul@iucaa.ernet.in \\ Accepted for publication: February 02, 2014
}

\begin{abstract}
Cosmological and astronomical observations predict that the present Universe is passing through an accelerating phase of expansion. The Universe emerged out of an exponential phase in the very early Universe. The scalar field of the standard model of particle physics when used in cosmology admits such a phase of expansion known as inflation. The most favourable condition for inflation with scalar field to admit an Inflationary scenario is that the potential energy must dominate over the kinetic energy which one obtains with a flat potential. Thereafter the Universe enters into a matter dominated phase when the field oscillates at the minimum of the potential. But it is not possible to accommodate the present accelerating phase in the Einstein's gravity. It is known from observational analysis that about $73 \%$ matter is responsible for the late phase expansion and $23 \%$ matter called Dark Matter is responsible for a stable galaxy. We discuss here the relevant fields and theories that are useful for describing the late Universe.
\end{abstract}

(C) 2014 RCOST: All rights reserved.

Keywords: Dark Matter; Dark Energy; Stable galaxy; Late Universe.

\section{Introduction}

In 1915, Einstein proposed the General theory of Relativity (GTR, henceforth) which is important in understanding the origin and evolution of the Universe [1- 4]. GTR is also important to understand highly massive objects where the strength of gravity is not weak. Einstein applied his famous equation in 1917 to obtain a model of the Universe which is static but failed to achieve a static model of the Universe with the field equation. This is because of the limitation of the then astronomical instruments which could observe only upto the periphery of our galaxy (Milkyway) i.e., up to a region which is almost not changing. Einstein then modified his GTR field equation by introducing a term which corresponds to repulsive force called cosmological constant. In 1927, while studying spectra of galaxies Hubble discovered that the Universe is expanding. It may be pointed out here that existence of non-static solution of the Einstein's field equation was noted by Friedmann in 1917. But those solutions were of academic interest for more than ten years. Hubble's discovery led Einstein to give away the cosmological constant term from the GTR equation. Hubble's discovery also supports the fact that the Universe originated from a Big-Bang in the past from a cosmic egg. The Universe was very hot after its birth in the Big-Bang model. As it

\footnotetext{
* Invited Speaker

International Conference on Emerging Trends in Science and Technology (March 22-23, 2014) Biratnagar, Nepal
} 
evolved, the temperature of the Universe decreased due to adiabatic expansion. The nucleosynthesis of the Universe might have occurred in the first three minutes. Subsequently stars, galaxies and the large scale structure of the Universe came up. After the discovery of Cosmic Microwave Background Radiation [5] the Big-Bang model became the standard model of the Universe which has a beginning of the Universe at some finite past.

However, Big-Bang model based on perfect fluid model failed to account some of the observed facts of the Universe. While probing the early Universe the problems that cropped up are the horizon problem, flatness problem, singularity problem. However, the above problems can be addressed in the framework of standard model of particle physics by invoking the concept of inflation [1-4, 6-12]. The matter is replaced by scalar fields and the necessary condition for inflation may be achieved successfully. The small quantum fluctuation of the fields that originated in the very early Universe leads to the structure formation of the Universe [3]. It is further known from the study of galaxy rotation curve that in addition to visible matter another form of matter known as Dark Matter exists in nature.

Recent astronomical and cosmological observations predict existence of a new form of energy hitherto unknown. The discovery of Riess et al. in 1998 [13] and Perlmutter et al. [15] in 1999 changed the landscape of vision of the observed Universe in addition to other cosmological observations. It is suggested that the current Universe is accelerating. In most of the investigations the origin of the acceleration is attributed to a mysterious component due to a source possessing negative pressure called Dark Energy (DE). Not only Type Ia supernova (SN Ia) observations but also cosmic microwave background (CMB) and baryon acoustic oscillations (BAO) have continued to confirm that about $73 \%$ of the energy density of the present Universe consists of Dark Energy. Dark Energy is a major outstanding issue in physics and cosmology today. In the literature the reported work mainly focused on theory, probes $\mathrm{DE}$ and on cosmological constant $[7,8,16]$. The preferred candidate for DE is a cosmological constant $\Lambda$. Consequently $\Lambda$ cold-dark-matter (CDM) model is constructed which plays the role of a model for alternative approaches to understand the DE-problems. Although $\Lambda \mathrm{CDM}$ fits most observational data well, it suffers from two main shortcomings: (i) the low value of the vacuum energy and (ii) the cosmic coincidence problem. In order to address the above issues a constant $\Lambda$ is replaced by a time varying $\Lambda$ resulting in a dynamical DE models. From cosmological observations it is also understood that about $23 \%$ matter in the Universe is in the form of Dark Matter (DM). In most investigations DM and DE are considered independent substance. But there is a possibility of existing coupling between DM and DE. So far DM and DE manifests only through their gravitational action. As a result the motivation for unified models of the cosmological substratum in which one single component plays the role of DM and DE simultaneously is studied. Recently, holographic principle [17] is incorporated in cosmology $[18,19]$ to track the Dark Energy content of the Universe following the work of Cohen et al [20]. In this paper we discuss the origin of Dark Matter and Dark Energy in the Universe and possible candidates in favour of DM and DE.

\subsection{Dark Matter in the Galaxies}

In 1932, a Dutch astronomer Jan Oort was the first to interpret evidence and infer the presence of Dark Matter. He found that the mass in the galactic plane must be more than the material that could be seen while studying stellar motions in the local galactic neighbourhood but this measurement was later determined to be essentially erroneous. In 1933, the Swiss astrophysicist Fritz Zwicky, who studied clusters of galaxies made a similar inference. Zwicky applied the virial theorem to the Coma cluster of galaxies and obtained evidence of unseen mass. In Fig.1, the observed galaxy rotation curve for M33 is shown. Let us consider an object (say a planet) at a distance $r$ from the gravitating centre (the Sun). The gravitational force on it is proportional to the product of masses and inversely proportional to the square of the distance. For dynamical equilibrium we determine the velocity of rotation of the planet. In the box below we consider solar system to evaluate rotational velocity of the planets. 
As an example in the above, we consider earth and a distant planet Neptune to estimate their rotational velocities. The values obtained using the Newton's formula is reliable. Now in the case of objects in the galaxies, analogously consider a light emitting source at a distance $r$ from the centre of the galaxy. For dynamical equilibrium the same velocity equation for the light emitting source will be valid and consequently velocity of rotation should fall as square root of the distance $r$ :

$$
v(r)=\sqrt{\frac{G M(r)}{r}} \propto \frac{1}{\sqrt{r}}
$$

where $\mathrm{M}(\mathrm{r})$ represents the mass which is supposed to be constant as was found in the case of a star in the solar system.

- For dynamical equilibrium

$$
\begin{aligned}
& \frac{G M m}{r^{2}}=\frac{m v^{2}}{r} \\
& \Rightarrow v=\sqrt{\frac{G M}{r}} \\
& r_{E}=150 \times 10^{6} \mathrm{Km} \Rightarrow v_{\text {earth }}=30 \mathrm{Km} / \mathrm{s} \\
& r_{N}=30 r_{E} \Rightarrow V_{\text {Neptune }}=5.4 \mathrm{Km} / \mathrm{s}(\text { reliable formula })
\end{aligned}
$$

Figure 1: Velocity of rotation

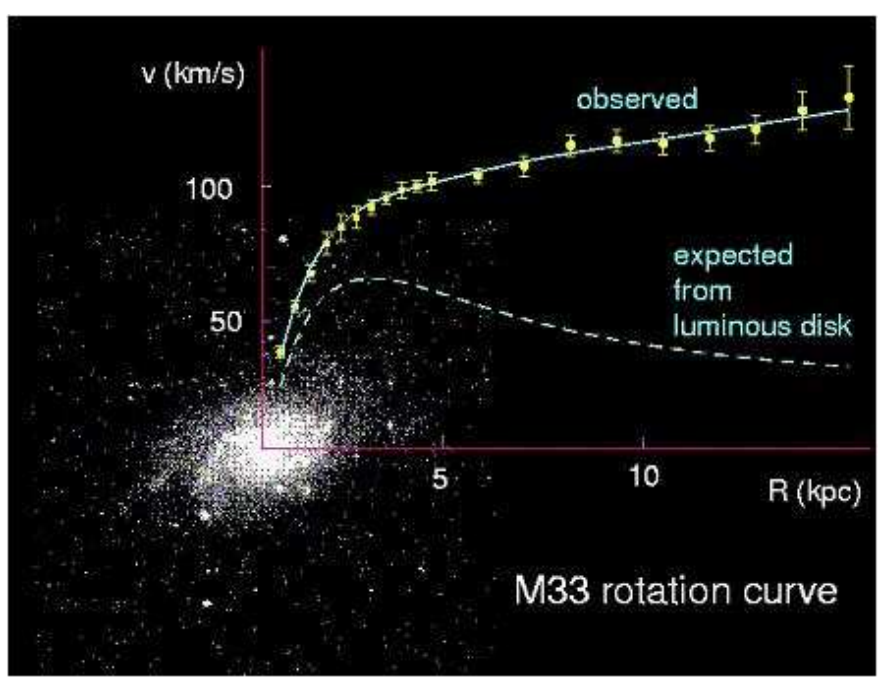

Figure 2: Rotation curve 
B.C. Paul / BIBECHANA 11(1) (2014) 8-16: (Online Publication: March, 2014) p.11

Now let us consider a galaxy consisting of about $10^{11}$ stars of mass equal to or more than the mass of the sun. Compare to the centre of the galaxy the stars are like point objects. In the case of M33 galaxy the observed velocity rotation curve as shown in Fig.(2), at first increases, it attains a maximum and thereafter its change in velocity is almost constant. But the expected velocity rotation curve from luminous disk after certain distance from the centre of the galaxy should decrease as shown. It is observed that

- Velocity at a distance $\mathrm{r}$ (denoted by $\mathrm{v}(\mathrm{r})$ ) rises towards a constant after about $2 \mathrm{kpc}$.

- $\mathrm{M}(\mathrm{r})$ interior to $r$ increases linearly with $\mathrm{r}$

Thus the above confrontation with observation may be removed assuming that the disc galaxies are immersed in extended Dark Matter (DM) halos, at small distances this DM is only a small fraction of the galaxy mass inside; it becomes very large at large distances. Let us estimate the Dark Matter from the following data: velocity of a luminous object at $10 \mathrm{kpc}$ away is $120 \mathrm{~km} / \mathrm{s}$ but its luminous velocity expected is $40 \mathrm{~km} / \mathrm{s}$. Using Eq. (1), we get

$$
\frac{M_{\text {total }}}{M_{\text {lum }}}=\frac{v_{T}^{2}}{v_{\text {Lum }}^{2}}
$$

where $\mathrm{M}_{\text {total }}$ represents the total mass and $\mathrm{M}_{\text {lum }}$ represents the luminous mass corresponding to rotational velocity $\mathrm{v}_{\mathrm{T}}$ and $\mathrm{v}_{\mathrm{lum}}$ respectively. We have the following data:

$$
\begin{aligned}
& r=10 \mathrm{kpc}, \quad v=120 \mathrm{~km} / \mathrm{s}, \quad v_{\text {lum. }}=40 \mathrm{~km} / \mathrm{s} \\
& \text { Thus }, \quad \frac{M_{\text {total }}}{M_{\text {lum }}}=\frac{v_{T}^{2}}{v_{\text {Lum }}^{2}}=\left(\frac{120}{40}\right)^{2}=9 \\
& \Rightarrow M_{\text {total }} \approx 9 M_{\text {lum }} \\
& \Rightarrow 90 \% \text { of mass in galaxies is DARK. }
\end{aligned}
$$

Thus a major composition of matter in the galaxies is Dark Matter.

\subsection{Dark Energy and the Dark Matter in Cosmology}

Cosmology is the study of the cosmos consisting of galaxies, clusters, superclusters etc. Einstein's general theory of relativity (GTR) is useful to understand the dynamics of the Universe. About 99 years back Einstein obtained field equation connecting geometry in one side and matter in the other side. This is an intuitive concept to relate the effect of matter in the space time. The space time geometry is flat in the absence of matter, however, in the presence of matter flat geometry changes its shape and it becomes curved. This curvature and matter are interrelated. The famous Einstein equation is

$$
\mathrm{R}_{\mu \nu}-\frac{1}{2} \mathrm{~g}_{\mu \nu} \mathrm{R}=-8 \pi \mathrm{G} \mathrm{T}_{\mu \nu}
$$

where $\mathrm{R}_{\mu \nu}$ and $\mathrm{R}$ represent the Ricci tensor and Ricci scalar respectively, $\mathrm{g}_{\mu v}$ represents the metric tensor, $\mathrm{T}_{\mu v}$ represents the energy momentum tensor and $\mathrm{G}$ is the Newton's gravitational constant. According to cosmological principle the Universe is isotropic and homogeneous and the most acceptable metric is the Robertson-Walker metric which is given by 


$$
d s^{2}=-d t^{2}+a^{2}(t)\left(\frac{d r^{2}}{1-k r^{2}}+r^{2}\left(d \theta^{2}+\sin ^{2} \theta d \varphi^{2}\right)\right)
$$

in the $(t, r, \theta, \phi)$ coordinates, $a(t)$ represents the scale factor of the Universe. In the above $k$ is the index which represents a closed Universe for +1 , open Universe for -1 and a flat Universe for 0 . In the case of perfect fluid the energy momentum is given by

$$
T_{\mu \nu}=[\rho,-p,-p,-p]
$$

where $\rho$ and p represent the energy density and pressure respectively. Using Eqs. (3) and (4) in Eq. (2) one obtains the field equations

$$
\begin{gathered}
3\left(\frac{a^{2}}{a^{2}}+\frac{k}{a^{2}}\right)=8 \pi G \rho \\
2 \frac{\ddot{a}}{a}+\left(\frac{a^{2}}{a^{2}}+\frac{k}{a^{2}}\right)=-8 \pi G p
\end{gathered}
$$

The famous Raychaudhuri Equation is obtained using Eqs. (5) and (6) as

$$
\frac{\ddot{a}}{a}=-\frac{4 \pi G}{3}(\rho+3 p)
$$

In 1926, Hubble discovered that the Universe is expanding, for which one requires to have matter with negative pressure as

$$
p<-\frac{\rho}{3}
$$

which is not permitted classically in the framework of perfect fluid assumption. Note that even $10^{-6} \mathrm{sec}$ after Big-Bang, $\mathrm{E}_{\text {(particle) }}>1 \mathrm{GeV}$. At this energy scale it is necessary to describe matter by quantum fields. For energies less than Planck energy, classical description of space-time possible with scalar fields permitted by Particle Physics. As discussed above the concept of inflation in cosmology is therefore essential which is permitted if quantum fields are considered. In 1981 Guth [6] proposed inflationary Universe scenario using temperature dependent phase transition mechanism in order to get rid of the problems of the Big-Bang cosmology. In this scenario there was a phase of expansion of the Universe when a small causally coherent region grows to a huge size to encompass the present Universe. Subsequently it is found that a graceful exit from inflation is not permitted in the scenario [7]. A new inflation is then proposed by Linde [8], Albrecht and Steinhardt [9, 10] where the graceful exit is natural. The period of inflation was very short in which the Universe grows rapidly so that at a later epoch it allows the different parts we see to come in casual contact with each other, thereby explaining the isotropy of the observed Universe. Eventually, the quantum vacuum state decays, dumping its energy into the form of thermal radiation and the Big-Bang Friedmann model takes over. In 1983, Linde [8] proposed a suitable inflationary Universe model which does not require temperature dependent phase transition mechanism instead the Universe can be realized from a chaotic distribution of a homogeneous scalar field. In this scenario sufficient inflation required to solve the problems of the Big-Bang model can be realized if a causally coherent region grow out with an initial scalar field which picks up values $\phi>3 \mathrm{M}_{\mathrm{P}}$, where $M_{P}$ represents the Planck mass. Quantum gravity region is not important in this case as $V(\phi) \geq M_{P}{ }^{4}$. Linde [8] has shown that a kinetic energy dominated region can pass on to an inflationary era afterwards as the potential energy domination sets in at a late epoch in scalar field cosmology. Paul et al. [11] shown 
that chaotic scenario is more realistic as it can be accommodated even in the presence of an anisotropy. An inflationary Universe model is important as it opens up new avenues not only in Cosmology but also in Particle Physics. It can solve some of the outstanding conceptual issues in cosmology Particle Physics not understood before. In the last three decades a number of inflationary models of the Universe proposed in the literature in the context of different theories e.g.

- (1980-1989): $\mathrm{R}^{2}$-inflation [12], Old inflation [6], New inflation [16], Chaotic inflation [8,21], Power-law inflation [22], Extended inflation [23], SUGRA inflation [24],Double inflation [25].

- (1990-1999): Hybrid inflation [26], SUSY D-term inflation [27], Brane inflation [28], Assisted inflation [29] .

- (2000-2008): Super-natural inflation [30], K-inflation [31], D3-D7 inflation [32], Tachyon inflation [33], Hill top inflation [34], DBI inflation [35].

Till now it is not clear when and how the Universe entered the inflationary phase in the past. A suitable model which can describe the entire evolution of the Universe is yet to be constructed. A baby Universe i.e., a tiny causally connected region grew into a huge size due to early inflation which engulfed the entire Universe in a very short time. During the creation the Universe was very hot as it expands its temperature decreases enhancing the formation of atoms from a soup of quark-gluon plasma. A photon thus emitted at very high temperature is the sole spectator of the phase which is commoving with the stratum. Its temperature decreases and the present $2.73 \mathrm{~K}$ black body radiation is due to this decoupled photon and expansion of the Universe. The frequency range of this radiation lies in the Microwave region at the present epoch which is showering isotropically in the Universe. This is cosmic Microwave background radiation (CMBR). Thus it is important to encode the message hidden in CMBR. Penzias and Wilson discovered the presence of CMBR while scanning the entire sky for source emitting microwave. Later they were awarded with nobel prize. In the inflationary theory the quantum fluctuations that developed in the early Universe leads to large structure formation which was supported by Cosmic Background Explorer (COBE). The present decade is witnessing a transition from speculative science to an experimental science because of a number of precision observational projects namely, CMB, SDSS, WMAP and PLANCK. The analysis of the data from those missions are confronting with theoretical cosmology. When observational data are analyzed in the framework of Big-Bang cosmology it comes out that the present Universe is composed of $73 \%$ Dark Energy (darkshade), $23 \%$ Dark Matter (violet) and only $4 \%$ (red) are baryons that is visible around us. A pie chart reflects the energy budget of the Universe.

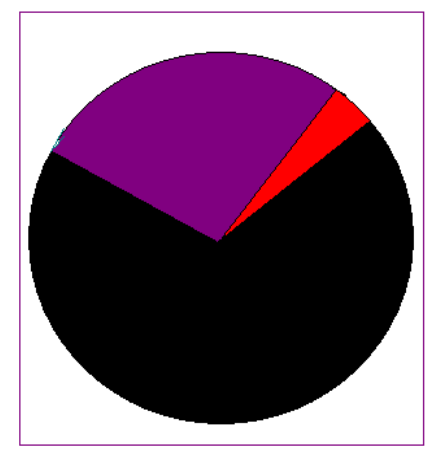

Figure 3: Energy distribution in the Universe Dark Energy (darkshade), Dark Matter (violet) and only $4 \%$ (red) observed matter. 
One of the most exciting observational discoveries of the past decade is that the Universe is accelerating. The source responsible for Cosmic Acceleration is presently unknown and has been called Dark Energy. Dark Energy has large negative pressure and could account for up to $73 \%$ of the total matter density in the Universe!!

Using eq. (5) with a number of fluids and defining the density parameter $(\Omega)$, and critical density $\left(\rho_{\mathrm{c}}\right)$ which represents the energy density corresponding to a flat Universe as below one obtains

$$
\begin{array}{r}
H=\frac{\dot{a}}{a}, \quad \Omega=\frac{\rho}{\rho_{c}}, \quad \rho_{c}=\frac{3 H^{2}}{8 \pi G} \\
\Rightarrow 1+\frac{k}{a^{2} H^{2}}=\frac{8 \pi G \rho}{3 H^{2}}=\frac{\sum \rho_{i}}{\rho_{c}}=\sum \Omega_{i} \\
\quad \Omega=\sum \Omega_{i} \\
\Rightarrow \Omega-1=\frac{k}{a^{2} H^{2}}
\end{array}
$$

In the case of rapid expansion in a very short period called inflation, through which the Universe emerged in the past, we get

$$
\begin{aligned}
& \Omega-1 \rightarrow 0 \quad \Rightarrow \Omega=\sum_{i} \Omega_{i} \approx 1 \\
& \therefore \quad \Omega_{\text {Lum }}+\Omega_{\text {nonLum }} \approx 1 \\
& \Omega_{\text {obs }} \approx 0.2 \Rightarrow \Omega_{\text {nonLum }} \approx 0.8
\end{aligned}
$$

For a number of fluids, we can write as

$$
\begin{aligned}
& \sum_{i=1}^{N} \Omega_{i}=1 \\
& \Rightarrow \Omega_{1}+\Omega_{2}+\Omega_{3}+\ldots=1 \\
& \Omega_{1}=0.2, \quad \Omega_{2}, \ldots
\end{aligned}
$$

Thus it is evident that the observation predicts existence of matter other than the matter we observe around us. It is predicted from supernova experiments that the Universe is expanding at a rate much faster than that theoretically calculated. This result is amazing!

In the Large Scale Structure (LSS) experiments dedicated telescope measured cosmological red shift of more than 2,50,000 galaxies! The derived galaxy-galaxy correlation function provides a tantalizing glimpse of the power spectrum of galaxy clustering and places powerful constraints on the density in clustered matter. It is also known from High Red shift type I a supernova study that the Universe is accelerating and its acceleration is fuelled by unknown form of Dark Energy having negative pressure.

Thus due to advancement of technology the present era is witnessing a transition of cosmology from speculative science to experimental science. A number of astronomical and cosmological observations in the recent past made it possible to visualize the Universe which is different from what we understood a decade ago. Riess et al. [13] and Perlmutter et al. [14] found that the present Universe is accelerating which they observed while analyzing data from large red shift survey of supernovae. This late acceleration cannot be realized in the framework of standard model of particle physics. It is one of the challenges in theoretical physics to develop a consistent theory to address the issue. It is found that old cosmological constant term of Einstein again can help to obtain such late accelerating Universe. However, origin of a cosmological constant term required at late epoch is not known. When observational results 


\section{B.C. Paul / BIBECHANA 11(1) (2014) 8-16: (Online Publication: March, 2014) p.15}

are analyzed in the Big-Bang model, existence of a new kind of energy that fills the space is required which is termed as Dark Energy. It has a negative pressure and negative gravitational effect causing a gravitational repulsion. As the Universe expands, Dark Energy stays at nearly constant energy density and, as the matter in the Universe thins out, the Dark Energy begins to dominate. The repulsive effect of Dark Energy seems to guarantee that the Universe might continue to expand forever. It is now a great challenge to theoretical physicists to give a consistent model for the Dark Energy in addition to Dark Matter. In the Einstein field equation this may be achieved by a modification of the gravitational sector [36] or the matter sector [37]. The work in this direction is now in progress worldwide to unravel the mystery of the Universe. Emergent Universe model in a flat Universe is another interesting model of the Universe which can be obtained with a non-linear equation of state [38]. In the emergent Universe scenario the initial size of the Universe was large enough so that quantum gravity effect is not important. The horizon and flatness problems do not arise. In the EU scenario, the Universe evolves from a static phase in the infinite past into an inflationary phase at a later epoch. In the usual description with a scalar field it is shown that a Universe starts expanding from the above phase, later on smoothly joins with a stage of exponential expansion followed by standard reheating and then approaches the classical thermal radiation dominated era of the conventional Big-Bang cosmology. The matter in the Universe may be considered as a composition of three kinds of fluids depending on EoS parameter. In the model one of the compositions of matter is Dark Energy. It permits an accelerating Universe which is supported by observations.

\section{Acknowledgment}

The Author acknowledges the University Grants Commission, New Delhi for awarding a Major Research Project (Grant No. F.42-783/2013 (SR)).

\section{References}

1. S.W. Weinberg, Gravitation \& Cosmology, John Wiley and Sons, 2004.

2. J.V. Narlikar, Introduction to Cosmology, Cambridge University Press, 1993.

3. T. Padmanabhan, Theoretical Cosmology Vol. III, Cambridge University Press, 2002.

4. S. Dodelson, Modern Cosmology, Academic Press, 2003.

5. A.A. Penzias, R. W. Wilson, Astrophys. J., 142( 1965)419.

6. A.H. Guth, Phys. Rev. D, 23 (1981)347.

7. K. Sato, Mon. Not. Roy. Astron. Soc., 195 (1981) 467.

8. A.D. Linde, Phys. Lett. B, 108 (1982) 389.

9. A. Albrecht, P. Steinhardt, Phys. Rev. Lett., 48 (1982) 1220.

10. A. Albrecht, arXiv:astro-ph/0007247, 2000.

11. B.C. Paul, D.P. Datta, S. Mukherjee, \{lit Mod. Phys. Letts. A\} \{lbf 1 \}, 149 (1986).

12. A.A. Starobinsky, JETP Lett., 30 (1979) 682; A. A. Starobinsky, Phys. Lett. B, 91(1980)99.

13. A.G. Riess et al., Astron J., 116 (1998) 1009.

14. S. Perlmutter et al., Nature, 51 (1998)391.

15. S. Perlmutter et al., Astrophys. J., 517 (1999) 565.

16. A.H. Guth, E.J. Weinberg, Nucl. Phys. B, 212 (1983) 321.

17. W. Fishler, L. Sisskind, hep-th/9806039, Bousso R 1999 JHEP 9907, 004; Bousso R 2000 Class Quantum Grav., 17, 997.

18. S.D.H. Hsu, Phys. Lett. B, 3 (2004) 594.

19. M. Li, Phys. Lett. B, 1 (2004) 603.

20. A.G. Cohen, D.B. Kaplan, A.E. Nelson, Phys. Rev. Lett., 82 (1999) 4971.

21. A.D. Linde, Rep. Prog. Phys. 47 (1984) 925; A.D. Linde, Phys. Lett B,162 (1985) 281; A.D. Linde, Phys. Lett B, 129 (1983) 177.

22. L.F. Abbott, M.B. Wise, Nucl. Phys. B 244 (1984) 541; F. Lucchin, S. Matarrese, Phys. Rev. D, 32 (1985) 1316; J.D. Barrow, Phys. Lett. B, 187 (1987) 12; J. Yokoyama, K. Maeda, Phys. Lett. B, 207 (1988) 31; A.R. Liddle, Phys. Lett. B, 220 (1989) 502. 
B.C. Paul / BIBECHANA 11(1) (2014) 8-16: (Online Publication: March, 2014) p.16

23. D. La, P.J. Steinhardt, Phys. Rev. Lett., 62 (1989) 376; D. La, P.J. Steinhardt, Phys. Lett. B, 220 (1989) 375.

24. E.J. Copeland, A.R. Liddle, D.H. Lyth, E.D. Stewart, D. Wands, Phys. Rev. D, 49 (1994) 6410. M. Yamaguchi, Phys. Rev. D, 64 (2001) 063502.

25. A.D. Linde, Phys. Lett. B, 38 (1991) 259; A.D. Linde, Phys. Rev. D, 49 (1994) 748; D. Lyth, A. Riotto, Phys. Rep. 1 (1999) 314.

26. E. Halyo, Phys. Lett. B, 47 (1996) 387; P. Binetruy, G. Dvali, Phys. Lett. B, 388 (1996) 241.

27. P. Horava, E. Witten, Nucl. Phys. B, 460 (1996) 506; P. Horava, E. Witten, Nucl. Phys. B, 475 (1996) 94.

28. A.R. Liddle, A. Mazumdar, F.E. Schunck, Phys. Rev. D, 58 (1998) 061301; A. Jokinen, A. Mazumdar, Phys. Lett. B, 597 (2004) 222.

29. L. Randall, M. Soljacic, A.H. Guth, Nucl. Phys. B, 472 (1996) 377 .

30. C. Armendariz-Picon, T. Damour, V. Mukhanov, Phys. Lett. B, 458 (1999) 209.

31. K. Dasgupta, C. Herdeiro, S. Hirano, R. Kallosh, Phys. Rev. D, 65 (2002) 126002; F. Koyama, Y. Tachikawa, T. Watari, Phys. Rev. D, 69 (2004) 106001; J.P. Hsu, R. Kallosh, JHEP, 0404 (2004) 042; K. Dasgupta, J.P. Hsu, R. Kallosh, A. Linde, M. Zagermann, JHEP, 0408 (2004) 030; P. Chen, K. Dasgupta, K. Narayan, M. Shmakova, M. Zagermann, JHEP, 050 (2005) 009; G. Gibbons, Phys. Lett. B, 537 (2002) 1.

32. L. Boubekeur, D.H. Lyth, JCAP, 0507 (2005) 010; K. Kohri, C.M. Lin, D.H. Lyth, JCAP, 0712 (2007) 004.

33. J.E. Lidsey, I. Huston, JCAP, 0707 (2007) 002; W.H. Kinney, K. Tzirakis, Phys. Rev. D, 77 (2008) 103517.

34. S.M. Carroll, Living Rev. Rel., 4 (2001) 1; R.H. Dicke, P.J.E. Peebles, P.J. Roll, D.T. Wilkinson, Astrophys. J. Lett., 142 (1965) 414; S. Mukherjee, B.C. Paul, S.D. Maharaj, A. Beesham, arXive:grqc/0505103 (2005); B.C. Paul, S. Ghose, Gen. Rel. Grav., 42 (2010) 795; G.F.R. Ellis, R. Maartens, Class. Quant. Grav., 21 (2004) 223; E.R. Harrison, Mont. Not. Roy. Aston. Soc., 69 (1967) 137; G.F.R. Ellis, J. Murugan, C.G. Tsagas, Class. Quant. Grav., 21 (2004) 233; D.J. Murlryne, R. Tavakol, J.E. Lidsey, G.F.R. Ellis, Phys. Rev. D, 71 (2005) 123512; S. Mukherjee, B.C. Paul, N.K. Dadhich, S.D. Maharaj, A. Beesham, Class. Quant. Grav., 23 (2006) 6927; S. del Campo, R. Herrera, P. Labrana, J. Cosmo. Astropart. Physics, 30 (2007) 0711; A. Beesham, S.V. Chervon, S.D. Maharaj, Class. Quantum Grav., 26 (2009) 075017; S. Nojiri, S.D. Odintsov, Phys. Rev. D, 70 (2004) 103522; arXiv: hepth/0408170; S. Nojiri, S.D. Odintsov, Phys. Rev. D, 72 (2005) 023003; arXiv: hepth/0505215; J. D. Barrow, Nucl. Phys. B, 310 (1988) 743; J.D. Barrow, Phys. Lett. B, 235 (1990) 40; V. Gorini, A. Kamenshchik, U. Moschella, V. Pasquier, Phys. Rev. D, 69 (2004)123524, S. Chaplygin, Sci. Mem. Moscow Univ. Math. Phys. 21 (1904) 1; A. Kamenshchik, U. Moschella, V. Pasquier, Phys. Lett. B 511 (2001) 265; Z.H. Zhu, Astron. Astrophys., 423 (2004) 421;M.C. Bento, O. Bertolami, A.A. Sen, Phys. Lett. B, 575 (2003) 172; N. Bilic, G.B. Tupper, R.D. Viollier, Phys. Lett. B, 535 (2001) 17; M.C. Bento, O. Bertolami, A.A. Sen, Phys. Rev. D, 66 (2002) 043507; J.C. Fabris, P.L.C. de Oliveira, H.E.S. Velten, Eur. Phys. J. C, 71 (2011) 1773; L. Amendola, F. Finelli, C. Burigana, D. Carurans, JCAP, 0307 (2003) 005.

35. S. Mukherjee, B.C. Paul, N.K. Dadhich, S.D. Maharaj, A. Beesham, Class. Quant. Grav., 23 (2006) 6927; S. Mukherjee, B.C. Paul, S.D. Maharaj, A. Beesham, arXive:gr-qc/0505103 (2005); B.C. Paul, S. Ghose, Gen. Rel. Grav., 42 (2010) 795; A. Banerjee, T. Bandyopadhyay, S. Chakraborty, Gen. Rel. Grav. 40 (2008) 1603; A. Banerjee, T. Bandyopadhyay, S. Chakraborty, Grav. and Cosmology 13 (2007) 290; U. Debnath, Class. Quant. Grav., 25 (2008) 205019; B.C. Paul, P. Thakur, S. Ghose, Mon. Not. Roy. Astron. Soc., 407 (2010) 415; B.C. Paul, S. Ghose, P. Thakur, Mon. Not. Roy. Astron. Soc., 413 (2011) 686; S. Ghose, P. Thakur, B.C. Paul, Mon. Not. Roy. Astron. Soc., 421 (2012) 20. 